Pacific Journal of Mathematic 


\title{
POSITIVE SOLUTIONS AND SPECTRAL PROPERTIES OF SECOND ORDER ELLIPTIC OPERATORS
}

\author{
W. Allegretto
}

\begin{abstract}
Let $l$ denote a second order elliptic expression in divergence form and with coefficients defined in an exterior domain. In this paper it is shown that, under suitable conditions, the equation $l v=0$ has an a.e. positive generalized solution $v$ defined in a neighborhood of infinity. This is done under weaker conditions on the coefficients of $l$ than was previously required. It is then shown that the existence of such a $v$ implies the finiteness of the negative spectrum of operators naturally associated with $l$.
\end{abstract}

Let $G$ denote an exterior domain (open, connected set) of Euclidean $n$-space $E^{n}$ with smooth finite boundary (if any). As customary, we let $x=\left(x_{1}, \cdots, x_{n}\right)$ denote a point of $E^{n}$ and set $D_{i}=\partial / \partial x_{i}$ for $i=$ $1, \cdots, n$. Let $l$ denote the formal symmetric elliptic expression defined by:

$$
I u=-\sum_{i, j=1}^{n} D_{i}\left(a_{i j} D_{j} u\right)+q u .
$$

The coefficients of $l$ are assumed real, and defined in $G$.

Some time ago, [2], we showed that if the coefficients of $l$ were assumed sufficiently regular and if $l$ was nonoscillatory (at $\infty$ ) then there was a positive function $v$ of class $C^{2}$ such that $\mathfrak{l} v \equiv 0$ near $\infty$. Recently, [7], Moss and Piepenbrink obtained, by different methods, the existence of such a positive solution for a more general expression in nondivergence form, with the coefficients now assumed to be only locally Hölder continuous. The interest in showing the existence of such a function stems from the connection between the existence of a positive solution and the spectral properties of operators associated with expression (1). We refer the reader to [8], [9] where this connection is described.

It is the purpose of this paper to extend the above results to cover cases where the coefficients of $l$ are less regular than was previously assumed. Our assumptions will be more in keeping with the requirements usually placed on the coefficients in spectral theory problems, see for example, [10]. Our basic method will consist in the extension and improvement of the ideas introduced in [2]. Consequently, we make use of quadratic forms and Hilbert space theory, as opposed to the Schauder estimate approach used in [7].

We remark that to simplify notation, different constants, whose 
precise value is irrelevant to the results, will be denoted by the same symbol. The same procedure will be applied to subdomains and subsequences. Further, we will not distinguish in notation between functions and equivalence classes to which such functions may belong. Finally we observe that an analogous presentation can be made for the case of an arbitrary unbounded domain or the case of a bounded domain with singularities on the boundary, (see, for example, [3]). Consequently, we do not pursue this further here.

Let $S$ denote a subdomain of $G, S \subseteq G$. We introduce the form $B(\phi, \psi, S)$ defined by the expression:

$$
B(\phi, \psi, S)=\int_{S} \sum_{i, j=1}^{n} a_{i j} D_{i} \phi D_{j} \psi+q \phi \psi,
$$

on pairs of locally integrable functions $\langle\phi, \psi\rangle$, with locally integrable generalized derivatives $D_{i} \phi, D_{i} \psi$, and for which the expression in (2) is finite. Here and in the sequel we shall always assume that: $a_{i j}=a_{j i}$ a.e. $G$; the matrix $\left(a_{i j}\right)$ is a.e. uniformly positive definite in any bounded subdomain of $G ; a_{i j}, q$ are of class $L_{100}^{1}(G)$ for $i, j=$ $1, \cdots, n$. We also make the basic assumption that the form $B$ is nonoscillatory. That is, there is neighborhood $N$ of $\infty$ such that for any bounded subdomain $P$ of $N$ there exists a constant $K=K(P)>0$ such that:

$$
B(\phi, \phi, P) \geqq K(\phi, \phi)
$$

for any $\phi \in C_{0}^{\infty}(P)$, where (, ) denotes the $L^{2}$ inner product. This definition is in direct analogy with the standard definition of the nonoscillation of an operator or expression. A survey of results connected with this terminology and subject may be found in the books of Swanson [12], and Kreith [6].

We next let $H_{1, p}(S), \stackrel{\circ}{H}_{1, p}(S)$ denote the standard Sobolev spaces with associated norm:

$$
\|\boldsymbol{v}\|_{1, p}(S)=\left[\int_{S} \sum_{i}\left(D_{i} v\right)^{P}+v^{P}\right]^{1 / P} .
$$

In direct analogy, and following a well-known procedure, we then define for $S \subset G$ the form:

$$
B^{\prime}(v, \psi, S)=\int_{S} \sum a_{i j} D_{i} v D_{j} \psi+\left(q^{+}+1\right) v \psi
$$

where the domain of $B^{\prime}$ is assumed to be $E \times E$ where $E$ is the subset of $C^{1}(\bar{S})$ such that $v \in E$ iff $B^{\prime}(v, v, S$ ) (as given by (4)) is finite, and we denote by $W(S)$ (respectively $\dot{W}(S)$ ) the completion of $E$ (respectively $\left.C_{0}^{\infty}(S)\right)$ in the norm $\|v\|_{W}(S)=\left(B^{\prime}(v, v, S)\right)^{1 / 2}$. A func- 
tion $u \in L_{\text {loc }}^{2}(S)$ is then termed to be in $W_{\text {loc }}(S)$ iff it is in $W\left(S^{\prime}\right)$ for any bounded subdomain $S^{\prime} \subset \bar{S}^{\prime} \subset S$. If $S$ is clear from the context, we write $W$ for $W(S), B(u, v)$ for $B(u, v, S)$, etc.

We now formulate the following local assumptions concerning the form $B$ :

There exists a sequence of closed nested smooth surfaces $\left\{R_{t}\right\}_{t=1}^{\infty}$ such that for any $r>0$ we have $R_{\mathfrak{r}} \subset\{|x|>r\}$ for $\mathfrak{l}$ large; and such that if we let $U_{\mathfrak{l}, k}$ denote the domain bounded by $R_{\mathfrak{l}}$ and $R_{k}(k>\mathfrak{l})$ with, for convenience, $R_{0}=\partial G$ (if any) and $U_{\mathfrak{l}, \infty}=\bigcup_{j=\imath+1}^{\infty} U_{\mathfrak{r}, j}$, we then have:

(a) for each $\mathfrak{l}$ sufficiently large and $k>\mathfrak{l},\|\phi\|_{W}\left(U_{\mathfrak{l}, k}\right)$ is equivalent to $\left[B\left(\phi, \phi, U_{t, k}\right)\right]^{1 / 2}$ on $C_{0}^{\infty}\left(U_{t, k}\right)$.

(b) for each $k$ sufficiently large there exists a neighborhood $N_{k}$ of $R_{k}$ in which $a_{i j}, q$ are of class $L^{\infty}$ for $i, j=1, \cdots, n$ (we observe that, as a consequence, $\|v\|_{W}\left(N_{k}\right)$ is equivalent to $\left.\|v\|_{1,2}\left(N_{k}\right)\right)$.

(c) if $u \in W_{\text {loc }}\left(U_{\mathfrak{l}, \infty}\right)$ for some $\mathfrak{l}$, is such that $B(u, \phi)=0$ for all $\dot{\phi} \in C_{0}^{\infty}\left(U_{\mathfrak{l}, \infty}\right)$, then for each $k>\mathfrak{l}$ there exists a neighborhood $N_{k}$ of $R_{k}$ in which $u$ is of class $C^{1+\alpha}$.

(d) for $k, \mathfrak{l}$ sufficiently large, we can express $U_{k, \mathfrak{l}}$ in the form $U_{k, \mathfrak{l}}=G_{k, \mathrm{l}} \cup Z_{k, \mathrm{l}}$ where $Z_{k, \mathrm{l}}$ is a set of measure zero and $G_{k, \mathrm{l}}$ is a domain such that if $T$ is any proper subdomain of $G_{k, \text { r }}$ (i.e., $\bar{T} \subset G_{k, \mathrm{l}}$ ) and: $u \in W(T), u \geqq 0, B(u, \phi, T)=0$ for all $\phi \in C_{0}^{\infty}(T)$, then $u$ satisfies a Harnack condition in the proper subdomains of $T$ (i.e., ess $\sup _{x \in T^{\prime}} u \leqq$ $K \operatorname{ess} \inf _{x \in T^{\prime}} u$ for $\left.T^{\prime} \subseteq \bar{T}^{\prime} \subset T\right)$ and, further, either $u \equiv 0$ or $\operatorname{ess} \inf (u)>0$ in $T^{\prime}$.

(e) if for any given constant $c$ we define the form $B^{\prime \prime}$ by:

$$
B^{\prime \prime}\left(v, \psi, U_{0, k}\right)=\int_{U_{0, k}}\left(\sum a_{i j} D_{i} v D_{j} \psi+q v \psi\right) d x+\int_{R_{k}} c v \psi d s
$$

on $v, \psi$ of class $C^{1}\left(\overline{U_{0, k}}\right) ; v, \psi \equiv 0$ near $R_{0}$ if $R_{0} \neq \Phi$, then we assume that $B^{\prime \prime}\left(v, v, U_{0, k}\right)$ is nonnegative for any $v$ in the orthogonal complement of a finite dimensional subspace (depending on $k, c$ ) of $L^{2}\left(U_{0, k}\right)$, where if $\partial G=\Phi$ we denote by $U_{0, k}$ the bounded domain with boundary $R_{k}$.

We next identify $W\left(U_{\mathrm{r}, k}\right)$ as a subspace of $H_{1,2}\left(U_{\mathrm{t}, k}\right)$ and observe that if $v \in W\left(U_{\mathfrak{r}, k}\right)$ then

$$
\|v\|_{W}^{2}\left(U_{\mathfrak{r}, k}\right)=\int_{U_{\mathfrak{r}, k}} \sum a_{i j} D_{i} v D_{j} v+\left(q^{+}+1\right) v^{2}
$$

where $D_{i} v$ denotes the $H_{1,2}$ derivative of $v$ for $i=1, \cdots, n$. The same remark holds for the inner products $(u, v)_{W}, B\left(u, v, U_{t, k}\right)$ with $u, v \in W\left(U_{\mathrm{t}, k}\right)\left(\stackrel{\circ}{W}\left(U_{\mathrm{r}, k}\right)\right.$ respectively).

For the reader's convenience we state a set of explicit conditions 
on $\left(a_{i j}\right), q$ which are sufficient for (a), (c)-(e) to hold, and sketch a brief proof.

LEMMA 0. For the above conditions to hold it is sufficient that $n \geqq 3$ and:

(i) for each $k$ sufficiently large there is a neighborhood $N_{k}$ of $R_{k}$ such that $a_{i j} \in C^{1}\left(N_{k}\right), q \in L^{\infty}\left(N_{k}\right)$;

(ii) for each subdomain $T \subset \bar{T} \subset G_{k, 1}$ we have $\left|a_{i j}\right|<M, q \in L^{r / 2}$ in $T$ with $M, r$ constants (depending on $T$ ) and $r>n$;

(iii) $q^{-} \in L^{n / 2}\left(U_{0,1}\right)$ for each $\mathfrak{Y}$.

Proof. We recall that $\left(a_{i j}\right)$ is assumed to be locally uniformly positive definite a.e. $G$. If $q^{-} \in L^{n / 2}\left(U_{0, k}\right)$ then, as is well known, we may assume that $q^{-}=q^{\prime}+q^{\prime \prime}$ with $q^{\prime}$ bounded and with $q^{\prime \prime}$ of small $L^{n / 2}$ norm.

Consequently, for any $\phi \in C_{0}^{\infty}\left(U_{t, k}\right)$ we have, by a standard estimate, [5],

$$
\int q^{\prime \prime} \phi^{2} \leqq \varepsilon \int \sum a_{i j} D_{i} \phi D_{j} \phi
$$

where $\varepsilon$ is a small positive constant. From (3) and (5) it follows that:

$$
B\left(\phi, \phi, U_{\mathrm{t}, k}\right) \geqq(1-\varepsilon) B^{\prime}\left(\phi, \phi, U_{\mathrm{t}, k}\right)-\left(\sup \left|q^{\prime}\right|+1\right) K B\left(\phi, \phi, U_{\mathrm{t}, k}\right)
$$

for some constant $K$ and, therefore,

$$
B\left(\phi, \phi, U_{\mathfrak{t}, k}\right) \geqq \frac{(1-\varepsilon)}{1+K\left(\sup \left|q^{\prime}\right|+1\right)} B^{\prime}\left(\phi, \phi, U_{\mathfrak{t}, k}\right) .
$$

It is obvious that $B^{\prime}\left(\phi, \phi, U_{\mathfrak{i}, k}\right)$ exceeds $B\left(\phi, \phi, U_{\mathfrak{r}, k}\right)$. Consequently, condition (a) is satisfied. Next, by (i), it follows that $B\left(u, \phi, N_{k}\right)=0$ implies that $u \in H_{1, p} \cap H_{2,2}$ with $p>n$, in a neighborhood of $R_{k}$ (see [1], [5]). By considering the equation satisfied by $D_{i} u$ we see, [11], that $D_{i} u \in C^{\alpha}$ in this neighborhood for $i=1, \cdots, n$, and condition (c) follows. Again by [11] we see that (ii) implies that condition (d) holds. Finally, to see that condition (e) is satisfied, observe that:

$$
c \int_{R_{k}} v^{2} d s=c \int_{U_{0, k}} \sum_{i} D_{i}\left(v^{2} \xi_{i}\right) d x
$$

where $\xi_{i}$ is a regular function which extends $\cos \left(n, x_{i}\right)$ into $U_{0, k}$. Consequently, for any $\varepsilon>0$ we have for some constants $K_{\varepsilon}, c_{1}$,

$$
\int_{R_{k}} c v^{2} d s \leqq c_{1} \int_{U_{0, k}}\left\{v^{2}+\sum_{i}|v|\left|D_{i} v\right|\right\} d x
$$




$$
\begin{aligned}
& \leqq c_{1} \int_{U_{0, k}}\left\{v^{2}+\varepsilon^{-2}|v|^{2}+\varepsilon^{2} \sum_{i}\left|D_{i} v\right|^{2}\right\} d x \\
& =\varepsilon^{2}\|v\|_{1,2}^{2}\left(U_{0, k}\right)+K_{\varepsilon}\|v\|_{0,2}^{2}\left(U_{0, k}\right)
\end{aligned}
$$

where $\|v\|_{0, p}(S)$ denotes the $L^{p}(S)$ norm. It follows that, for some positive constants $C, C_{1}$, we have, by again estimating the $q^{-}$term as in $[5$, p. 27$]$,

$$
B^{\prime \prime}\left(v, v, U_{0, k}\right) \geqq C\left\{\|v\|_{1,2}^{2}\left(U_{0, k}\right)-C_{1}\|v\|_{0,2}^{2}\left(U_{0, k}\right)\right\} .
$$

Condition (e) easily follows from (6) by the Sobolev compact imbedding theorem of $H_{1,2}\left(U_{0, k}\right)$ into $L^{2}\left(U_{0, k}\right)$.

We observe that the above conditions allow the coefficients to have $L^{1}$ singularities at points of $Z_{k, \mathrm{l}}$. However the nature of $Z_{k, \mathrm{l}}$ is restricted, so that we do not allow, for example, $L^{1}$ singularities along a ray or along a sequence of closed surfaces tending to $\infty$.

It is convenient to obtain a variety of properties for functions belonging to the $W$-spaces. We thus state:

LEMMA 1. (1) $W\left(U_{\mathrm{t}, k}\right) \cap \stackrel{\circ}{1,2}_{1}\left(U_{\mathrm{t}, k}\right)=\stackrel{\circ}{W}\left(U_{\mathrm{t}, k}\right)$.

(2) If $u \in \dot{W}\left(U_{\mathrm{t}, k}\right)$ (resp. $W\left(U_{\mathrm{t}, k}\right)$ ) then so do $u^{+}, u^{-}$, and $|u|$.

(3) If $u_{1}, u_{2} \in W\left(U_{t, k}\right), B\left(u_{1}, \phi\right)=B\left(u_{2}, \phi\right)$ for all $\phi \in C_{0}^{\infty}\left(U_{t, k}\right)$ and $\left(u_{1}-u_{2}\right)^{+} \in \stackrel{\circ}{W}\left(U_{1, k}\right)$ then $B\left(\left(u_{1}-u_{2}\right)^{+},\left(u_{1}-u_{2}\right)^{+}\right)=0$.

(4) If $v \in W_{\mathrm{loc}}\left(U_{\mathfrak{t}, \infty}\right), v \geqq 0$ a.e. $U_{\mathfrak{t}, \infty}$ then $\phi(v+\varepsilon)^{-1} \in \dot{W}\left(U_{\mathfrak{t}, \infty}\right)$ for any $\varepsilon>0$ and $\phi \in C_{0}^{\infty}\left(U_{\mathfrak{r}, k}\right)$.

Proof of Lemma 1. (1) Clearly $\stackrel{\circ}{W}\left(U_{\mathfrak{t}, k}\right) \subset W\left(U_{\mathfrak{t}, k}\right) \cap \stackrel{\circ}{H}_{1,2}\left(U_{\mathfrak{r}, k}\right)$. Conversely, let $u \in W\left(U_{t, k}\right) \cap \stackrel{\circ}{H}, 2_{1}\left(U_{t, k}\right)$, and let $\phi \in C_{0}^{\infty}\left(U_{t, k}\right), 0 \leqq \phi \leqq 1$, $\phi \equiv 1$ except sufficiently near $R_{\mathfrak{r},}, R_{k}$. Next, let $u_{n} \in C^{1}\left(\overline{U_{\mathrm{t}, k}}\right)$ be a sequence such that $u_{n} \rightarrow u$ in \|\|$_{W}\left(U_{\mathrm{t}, k}\right)$. It follows that:

$$
\begin{aligned}
\left\|\phi u_{n}\right\|_{W}^{2}\left(U_{\mathfrak{t}, k}\right)= & \int_{U_{\mathfrak{t}, k}} \sum_{i, j} a_{i j} D_{i}\left(\phi u_{n}\right) D_{j}\left(\phi u_{n}\right)+\left(q^{+}+1\right) \phi^{2} u_{n}^{2} \\
= & \int_{U_{\mathfrak{l}, k}}\left(\sum_{i, j} a_{i j} D_{i} \phi D_{j} \phi\right) u_{n}^{2}+\int_{U_{\mathfrak{t}, k}} \sum_{i, j} a_{i j} D_{i}\left(\phi^{2} u_{n}\right) D_{j} u_{n} \\
& +\left(q^{+}+1\right) \dot{\phi}^{2} u_{n}^{2} \\
= & I_{1}+I_{2} .
\end{aligned}
$$

Steps (7) and (8) are really a first order form of an identity due to Picone (see [4], [12], [6]) which is very useful for our purposes. Next, we observe that

$$
\begin{aligned}
I_{2} & =\int_{U_{\mathfrak{l}, k}} \phi^{2}\left[\sum_{i, j} a_{i j} D_{i}\left(u_{n}\right) D_{j}\left(u_{n}\right)+\left(q^{+}+1\right) u_{n}^{2}\right]+\int_{U_{\mathfrak{l}, k}} 2 \phi u_{n} \sum a_{i j} D_{i} \phi D_{j} u_{n} \\
& \leqq\left\|u_{n}\right\|_{W}^{2}+K\left(\int_{U_{\mathfrak{l}, k}} u_{n}^{2} \sum a_{i j} D_{i} \phi D_{j} \phi\right)^{1 / 2}\left(\int_{U_{\mathfrak{l}, k}} \phi^{2} \sum a_{i j} D_{i} u_{n} D_{j} u_{n}\right)^{1 / 2}
\end{aligned}
$$


Since on $\operatorname{supp}(\operatorname{grad} \phi)$ we have $a_{i j} \in L^{\infty}$, we conclude:

$$
I_{2} \leqq K\left\|u_{n}\right\|_{W}^{2} .
$$

A similar estimate is valid for $I_{1}$ and, consequently,

$$
\left\|\phi u_{n}\right\|_{W}^{2}\left(U_{\mathfrak{r}, k}\right) \leqq K\left\|u_{n}\right\|_{W}^{2}\left(U_{\mathfrak{r}, k}\right) \text {. }
$$

Since $\phi u_{n} \in C_{0}^{1}\left(U_{\mathrm{r}, k}\right)$ by substituting $u_{n}-u_{m}$ for $u_{n}$ in (9) it follows that $\phi u \in \dot{W}\left(U_{\mathfrak{l}, k}\right)$. Finally $(1-\phi) u$ has support near $R_{\mathfrak{\imath}}$ and $R_{k}$ where \|\|$_{1,2}$ is equivalent to \|\|$_{W}$. Consequently, $(1-\phi) u \in \stackrel{\circ}{H}_{1,2}\left(U_{t, k}\right) \cap$ $W\left(U_{\mathrm{r}, k}\right) \cap \dot{W}\left(U_{\mathrm{t}, k}\right)$, and $u=(1-\phi) u+\phi u \in \dot{W}\left(U_{\mathrm{t}, k}\right)$.

(2) Let $\phi \in C_{0}^{\infty}\left(U_{\mathrm{l}, k}\right)$ then $\phi^{+} \in \stackrel{\circ}{H}, 2_{1,}\left(U_{t, k}\right)$ (see, for example, [11]). Let $\psi_{n}$ be a sequence of mollifiers, $\psi_{n} \rightarrow \phi^{+}$in $H_{1,2}\left(U_{t, k}\right)$. Then $\left|D_{i} \psi_{n}\right| \leqq$ $\sup \left|D_{i} \phi\right|$ and $\left|\psi_{n}\right| \leqq \sup |\phi|$. It follows that:

$$
\begin{aligned}
\left\|\psi_{n}\right\|_{W}^{2}\left(U_{\mathfrak{r}, k}\right) & \leqq\left\{\int_{U_{\mathfrak{r}, k}}\left(\sum_{i, j}\left|a_{i j}\right|\right)\left[\sup \left|D_{j} \phi\right|\right]^{2}+\int_{U_{\mathfrak{l}, k}}\left(q_{+}+1\right) \sup \phi^{2}\right\} \\
& \leqq K .
\end{aligned}
$$

Without loss of generality, we conclude that $\left\{\psi_{n}\right\}$ is weakly convergent. By the theorem of Banach-Saks, we may assume that $\left\{(1 / n) \sum_{i=1}^{n} \psi_{i}\right\}$ is strongly convergent in $W\left(\right.$ to $\left.\phi^{+}\right)$. Consequently, $\phi^{+} \in \stackrel{\circ}{W}\left(U_{\mathrm{r}, k}\right)$, and,

$$
\begin{aligned}
\left\|\phi^{+}\right\|_{W}^{2}\left(U_{\mathfrak{r}, k}\right) & =\int_{U_{1, k}} \sum a_{i j} D_{i} \phi^{+} D_{j} \phi^{+}+\left(q^{+}+1\right)\left(\phi^{+}\right)^{2} \\
& \leqq\|\phi\|_{W}^{2}\left(U_{\mathfrak{r}, k}\right)
\end{aligned}
$$

Finally, if $\phi_{n} \rightarrow u$ in $\stackrel{\circ}{W}\left(U_{\mathfrak{r}, k}\right)$ then

$$
\left\|\left(\phi_{n}\right)^{+}\right\|_{W}^{2}\left(U_{\mathrm{r}, k}\right) \leqq\left\|\phi_{n}\right\|_{W}^{2}\left(U_{\mathrm{t}, k}\right) \leqq K
$$

Repeating the above procedure, we find that $u^{+} \in \dot{W}\left(U_{\mathrm{r}, k}\right)$, and by the same proof, the same is true of $u^{-},|u|=u^{+}+u^{-}$. The same argument shows the result for $u \in W\left(U_{\mathrm{r}, k}\right)$ once we observe that if $\phi \in C^{1}\left(\overline{U_{\mathrm{I}, k}}\right)$ then $\phi$ can be considered to be the restriction of a $C_{0}^{1}\left(U_{\mathfrak{l}-1, k+1}\right)$ function $\psi$. We conclude that $\phi^{+} \in W\left(U_{\mathfrak{l}, k}\right)$, and consequently that $\left\|\phi^{+}\right\|_{W} \leqq\|\phi\|_{W}$, and the result follows.

(3) Let $u_{1}, u_{2} \in W\left(U_{\mathrm{r}, k}\right)$ and set $u=u_{1}-u_{2}$. It follows that $B(u, \phi)=0$ for all $\phi \in C_{0}^{\infty}\left(U_{\mathfrak{r}, k}\right)$. Since by assumption we have $u^{+} \epsilon$ $\stackrel{\circ}{W}\left(U_{\mathrm{t}, k}\right)$ we conclude that

$B\left(u, u^{+}\right)=0$, i.e., that $B\left(u^{+}-u^{-}, u^{+}\right)=B\left(u^{+}, u^{+}\right)-B\left(u^{-}, u^{+}\right)=0$.

But $B\left(u^{-}, u^{+}\right)=0$ as a direct consequence of the fact that products of type $u^{+} u^{-}, D_{i} u^{+} D_{j} u^{-}$vanish almost everywhere by the very definition of $u^{+}, u^{-}$. It follows that $B\left(u^{+}, u^{+}\right)=0$. 
(4) Since $v=v^{+} \geqq 0$, by the mollifier procedure of (2), we construct a sequence $\left\{\psi_{m}\right\}$ of nonnegative $C^{1}(\operatorname{supp} \phi)$ functions such that $\psi_{m} \rightarrow v$ in $W(\operatorname{supp} \phi)$. Set $\beta_{m}=\phi\left(\psi_{m}+\varepsilon\right)^{-1}$. A direct calculation shows that:

$$
\begin{aligned}
\left\|\beta_{m}\right\|_{W}^{2}(\operatorname{supp} \phi) & \leqq K\left(\left\|\psi_{m}+\varepsilon\right\|_{W}^{2}(\operatorname{supp} \phi)+\|\phi\|_{W}^{2}\right) \\
& \leqq K\left(\|v+\varepsilon\|_{W}^{2}(\operatorname{supp} \phi)+\|\phi\|_{W}^{2}\right) .
\end{aligned}
$$

Again by the Banach-Saks theorem we conclude that $\phi(v+\varepsilon)^{-1} \epsilon$ $\stackrel{\circ}{W}\left(U_{\mathfrak{l}, \infty}\right)$.

CoRollary 1. (1) Let $u \in \stackrel{\circ}{W}\left(U_{\mathrm{t}, k}\right)$ be such that $B\left(u, \phi, U_{\mathrm{r}, k}\right)=$ $(f, \phi)$ for all $\phi \in C_{0}^{\infty}\left(U_{\mathrm{t}, k}\right)$ with $f \geqq 0$. Then $u \geqq 0$ in $U_{\mathrm{i}, k}$.

(2) Let $u_{i} \in \stackrel{\circ}{W}\left(U_{\mathrm{r}, k_{i}}\right)$ be such that $B\left(u_{i}, \phi, U_{\mathfrak{r}, k_{i}}\right)=(f, \phi)$ for all $\phi \in C_{0}^{\infty}\left(U_{\mathfrak{1}, k_{i}}\right)$ for $i=1,2$ and some $f \geqq 0$. If $k_{2} \geqq k_{1}$ then $u_{2} \geqq u_{1}$ in $U_{\mathrm{r}, k_{1}}$.

Proof. (1) Note that, as is well known, $u$ may be characterized as the minimizing function of:

$$
I(u)=B\left(u, u, U_{\mathfrak{r}, k}\right)-2(u, f) .
$$

Since $I(|u|) \leqq I(u)$, by Lemma 1 (2), we see that $u=|u| \geqq 0$.

(2) Observe that $\left(u_{1}-u_{2}\right)^{+} \in W\left(U_{\mathfrak{1}, k_{1}}\right) \cap \stackrel{\circ}{H}, 2_{1}\left(U_{1, k_{1}}\right)$. Consequently, by Lemma 1, $\left(u_{1}-u_{2}\right)^{+} \in \stackrel{\circ}{W}\left(U_{\mathfrak{r}, k_{1}}\right)$ and $B\left(\left(u_{1}-u_{2}\right)^{+},\left(u_{1}-u_{2}\right)^{+}\right)=0$. From our basic assumption it follows that $\left(u_{1}-u_{2}\right)^{+} \equiv 0$, i.e., $u_{2} \geqq u_{1}$.

LeMma 2. Let $k_{0}$ be chosen sufficiently large. Then there exists $r$ form $\widetilde{B}$ with coefficients $\tilde{a}_{i j}$, $\widetilde{q}$ such that: $\widetilde{a}_{i j}=a_{i j}, \widetilde{q}=q$ for $|x|$ sufficiently large; $\widetilde{a}_{i j}$, $\widetilde{q} \in C^{\infty}$ in a neighborhood $N_{k_{0}}$ of $R_{k_{0}}$; assumptions (a) to (d) hold for $\widetilde{B}$ on $U_{k_{0}-1, \mathfrak{l}}, \mathfrak{l}>k_{0}$.

Proof. Let:

$$
\begin{aligned}
\widetilde{a}_{i j} & =a_{i j}(1-\theta)+M \delta_{i j} \theta, \\
\widetilde{q} & =q(1-\theta)+N \theta,
\end{aligned}
$$

where:

$M=$ ess. sup. of the largest eigenvalue of $\left(a_{i j}\right)$ in $N_{k_{0}}$, $N=$ ess. sup. of $|q|$ in $N_{k_{0}}$, $\theta$ is a $C_{0}^{\infty}\left(N_{k_{0}}\right)$ function with $\theta \equiv 1$ near $R_{k}, 0 \leqq \theta \leqq 1$.

Now conditions (b), (c) are obvious. Condition (a) follows from the inequalities: 


$$
\begin{aligned}
\int_{U_{\mathfrak{l}, k}}(1-\theta) \sum a_{i j} D_{i} \phi D_{j} \phi & \leqq M \int_{U_{\mathfrak{t}, k}} \sum_{i}\left(D_{i} \phi\right)^{2} \leqq \frac{M}{\lambda} \int_{U_{\mathfrak{t}, k}} \sum a_{i j} D_{i} \phi D_{j} \phi \\
& \leqq \frac{M}{\lambda} B^{\prime}\left(\phi, \phi, U_{\mathfrak{t}, k}\right) \leqq \frac{M}{\lambda} C B\left(\phi, \phi, U_{\mathfrak{t}, k}\right) \\
& \leqq \frac{M}{\lambda} C \widetilde{B}\left(\phi, \phi, U_{\mathfrak{t}, k}\right) \\
\int_{U_{\mathfrak{t}, k}} N \theta \phi^{2} & \leqq N \int_{U_{\mathfrak{l}, k}} \phi^{2} \leqq \frac{N}{\lambda} B\left(\phi, \phi, U_{\mathfrak{t}, k}\right) \\
& \leqq \frac{N}{\lambda} \widetilde{B}\left(\phi, \phi, U_{\mathfrak{t}, k}\right) ;
\end{aligned}
$$

and:

$$
\begin{aligned}
\int_{U_{\mathfrak{l}, k}} q^{+}(1-\theta) \phi^{2} & \leqq \int_{U_{\mathfrak{l}, k}} q^{+} \phi^{2} \leqq B^{\prime}\left(\phi, \phi, U_{\mathfrak{t}, k}\right) \\
& \leqq C B\left(\phi, \phi, U_{\mathfrak{l}, k}\right) \leqq C \widetilde{B}\left(\phi, \phi, U_{\mathfrak{t}, k}\right),
\end{aligned}
$$

for some constants $\lambda, C$. Finally, condition (d) follows from the observation that if we set $k=\operatorname{dist}\left(\operatorname{supp} \theta, \partial N_{k_{0}}\right)$, then for any sphere $S$ with radius $\rho<k / 2$ we note that either $\bar{S}$ lies in $N_{k_{0}}$, in which case property (d) follows from the regularity of the coefficients, or else $\bar{S} \not \subset N_{k_{0}}$. In the latter case $\bar{S} \subset\{x \mid \theta(x)=0\}$ and property (d) follows from the original assumptions since in $\bar{S}$ the coefficients were not changed.

Note that if we can find a function $v$ such that $\widetilde{B}\left(v, \phi, U_{\mathrm{t}, \infty}\right)=0$ for all $\phi \in C_{0}^{\infty}\left(U_{\mathfrak{l}, \infty}\right)$ then $B\left(v, \phi, U_{\mathfrak{l}+1, \infty}\right)=0$ for all $\phi \in C_{0}^{\infty}\left(U_{\mathfrak{l}+1, \infty}\right)$. It follows that we may assume, without loss of generality, that $a_{i j}$ and $q$ are of class $C^{\infty}$ near any specific $R_{k}$.

We next recall the following lemma which was established in [2]:

LEMmA 3. Let $a_{i j} \in C^{\infty}\left(N_{k_{0}}\right), q \in C^{\infty}\left(N_{k_{0}}\right)$ for some neighborhood $N_{k_{0}}$ of $R_{k_{0}}$, and assume that $x_{0} \in N_{k_{0}}$. Then for all $\beta>0,0<\varepsilon<1 / 2$,

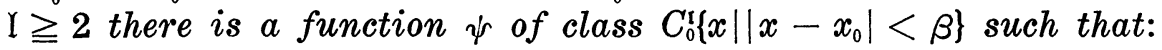

$$
\begin{array}{lll}
L \psi(x) \geqq 0 & \text { for } & \varepsilon \beta<\left|x-x_{0}\right|, \\
L \psi(x)>0 & \text { for } & \varepsilon \beta<\left|x-x_{0}\right|<(1-\varepsilon) \beta,
\end{array}
$$

where $L \psi=-\sum_{i, j} D_{i}\left(a_{i j} D_{j} \psi\right)+q \psi$.

THEOREM 1. Let $k_{0}$ be chosen sufficiently large so that the basic assumption holds for $U_{k_{0}-1, \infty}$. Then there exists a function $u \in$ $W_{\text {loc }}\left(U_{k_{0}+1, \infty}\right)$ such that: $B(u, \phi, \operatorname{supp} \phi)=0$ for any $\phi \in C_{0}^{\infty}\left(U_{k_{0}+1, \infty}\right)$; $u>0$ a.e. near $\infty$; for each $k$ sufficiently large there exists a neighborhood $N_{k}$ of $R_{k}$ such that $u \in C^{1+\alpha}\left(N_{k}\right)$. 
Proof. Without loss of generality, we may assume $a_{i j} \in C^{\infty}\left(N_{k_{0}}\right)$, $q \in C^{\infty}\left(N_{k_{0}}\right)$ in some neighborhood $N_{k_{0}}$ of $R_{k_{0}}$. Let $\psi$ be constructed by the procedure of Lemma 3 so that $L \psi \geqq 0(\not \equiv 0)$ in $U_{k_{0}, \infty}$ and $\operatorname{supp} \psi \subset N_{k_{0}}$.

We construct, as in [2], a sequence of functions $\left\{u_{j}\right\}_{j=k_{0}+1}^{\infty}$ such that $u_{j} \in \dot{W}\left(U_{k_{0}, j}\right)$ and $B\left(u_{j}, \phi\right)=(L \psi, \phi)$ for all $\phi \in C_{0}^{\infty}\left(U_{k_{0}, j}\right)$. By Corollary 1 we have: $u_{j+1} \geqq u_{j} \geqq 0$ and we set $u=\sup _{j} u_{j}$. The same procedure as in [2] shows that $u \in L_{10 \mathrm{co}}^{1}\left(M_{k_{0}} \cap U_{k_{0}, \infty}\right)$ where $M_{k_{0}}=N_{k_{0}}$ $\operatorname{supp} \psi$. Consequently, again see [2], the regularity of the coefficients in $N_{k_{0}}$ implies that $u \in C^{2}\left(M_{k_{0}} \cap U_{k_{0} \infty}\right)$ and, therefore, that $\left\{u_{j}\right\}$ is bounded in any proper subdomain of $M_{k_{0}} \cap U_{k_{0}, \infty}$. Since $u_{j}$ satisfies a Harnack inequality in the subdomains of $G_{k_{0}, k}$, by assumption (d), we conclude that $\left\{u_{j}\right\}$ is bounded near $R_{k}$ for any $k>k_{0}$ (with bound dependent on $k$ ). Now let $\phi \in C_{0}^{\infty}\left(U_{k, t}\right), 0 \leqq \phi \leqq 1$, supp $\phi \cap \operatorname{supp} L \psi=\Phi$, $\operatorname{supp}(\operatorname{grad} \phi)$ near $R_{k}, R_{t}$ for some $t, k, t>k>k_{0}$. Repeating the procedure of (7), (8) we find:

$$
\begin{aligned}
B\left(\phi\left(u_{m}-u_{n}\right), \phi\left(u_{m}-u_{n}\right)\right)= & \int_{U_{k, t}}\left(\sum a_{i j} D_{i} \phi D_{j} \phi\right)\left(u_{m}-u_{n}\right)^{2} \\
& +B\left(u_{m}-u_{n}, \phi^{2}\left(u_{m}-u_{n}\right)\right) \\
= & \int_{U_{k, t}}\left(\sum a_{i j} D_{i} \phi D_{j} \phi\right)\left(u_{m}-u_{n}\right)^{2}
\end{aligned}
$$

where we have used the fact that $\phi^{2}\left(u_{m}-u_{n}\right) \in \stackrel{\circ}{W}$ as was shown in the proof of Lemma 1(1). By the Lebesque convergence theorem applied to the righthand side of (10) and the fact that $\left\{u_{j}\right\}$ is bounded on $\operatorname{supp}(\operatorname{grad} \phi)$, we conclude that $\left\{\phi u_{j}\right\}$ is Cauchy in $W\left(U_{k, t}\right)$. Consequently, $u \in W_{\text {loc }}\left(U_{k, \infty}\right)$ and $B(u, \phi)=0$ for all $\phi \in C_{0}^{\infty}\left(U_{k, \infty}\right)$ as desired. Since $u \geqq u_{n} \geqq 0$ and $u_{n} \not \equiv 0$ then by assumption (d) it follows that $u>0$ a.e. in $U_{k, \infty}-\bigcup_{j=k+1}^{\infty} Z_{k, j}$ for $k$ sufficiently large. That is, $u>0$ a.e. in a neighborhood of $\infty$. Finally, $u \in C^{1+\alpha}\left(N_{k}\right)$ as a consequence of assumption (c).

CoROLlaRY 2. Let $v \in \dot{W}\left(U_{k_{0}+1, \mathfrak{l}}\right)$ for some $\mathfrak{l}>k_{0+1}, v \equiv 0$ outside $U_{k_{0}+1,1}$, then:

$$
\int_{U_{k_{0}, \infty}} \sum a_{i j} D_{i} u D_{j} v+q u v=0 .
$$

Proof. Let $\phi \in C_{0}^{\infty}\left(U_{k_{0}, \mathrm{r}+1}\right)$ now be such that $\phi \equiv 1$ in $U_{k_{0}+1, \mathfrak{l}}$ and $\operatorname{supp} \phi \cap \operatorname{supp} L \psi=\Phi$. Let $v_{n} \in C_{0}^{\infty}\left(U_{k_{0}+1, l}\right)$ tend to $v$ in $\stackrel{\circ}{W}$. Then:

$$
B(\phi u, v)=\lim _{n \rightarrow \infty} B\left(\phi u_{n}, v_{n}\right)=0 \text {, }
$$

but also:

$$
B(\phi u, v)=\int_{U_{k_{0}, \infty}} \sum a_{i j} D_{i} u D_{j} v+q u v
$$


THEOREM 2. There is a finite dimensional subspace $F \subset L^{2}(G)$ such that for all $\phi \in C_{0}^{\infty}(G) \cap F^{\perp}$ we have $B(\phi, \phi) \geqq 0$.

Proof. Let $\phi \in C_{0}^{\infty}(G)$ be given and choose $k_{0}$ sufficiently large, $\varepsilon>0$. Let $N_{k_{0}}$ be a neighborhood of $R_{k_{0}}$ and choose a $C_{0}^{\infty}$ function $\psi$ such that $\psi \equiv 1$ on $\overline{U_{0, k_{0}}}, \psi \equiv 0$ outside $U_{0, k_{0}} \cup N_{k_{0}}$. Let $u$ be the function constructed in Theorem 1 . Since $u$ is of class $C^{1+\alpha}$ near $R_{k_{0}}$ we may suppose $u$ to be extended as a nonnegative $C^{1+\alpha}$ function $\tilde{u}$ inside $R_{k_{0}}$ with $\tilde{u} \equiv 0$ outside of $U_{k_{0}, \infty} \cup N_{k_{0}}$. It follows that $\tilde{u} \in$ $W_{\text {loc }}\left(U_{0, \infty}\right)$ and therefore, by Lemma 0 , that $\phi^{2}(\tilde{u}+\varepsilon)^{-1} \in \dot{W}\left(U_{0, \infty}\right)$. We again employ the procedure of inequalities (7) and (8) and a limit argument to conclude:

$$
\int_{U_{k_{0}, \infty}} \sum a_{i j} D_{i} \phi D_{j} \phi+q \phi^{2} \geqq \int_{U_{k_{0}, \infty}} \sum a_{i j} D_{i}\left(\frac{\phi^{2}}{\widetilde{u}+\varepsilon}\right) D_{j}(\tilde{u}+\varepsilon)+q \phi^{2} .
$$

Setting $\phi^{2}=\phi^{2}(1-\psi)+\phi^{2}(\psi)$ on the right hand side of (11), observing that $\phi^{2}(1-\psi) /(\widetilde{u}+\varepsilon) \in \stackrel{\circ}{W}\left(U_{k_{0}, \infty}\right)$ and $u=\tilde{u}$ in $U_{k_{0}, \infty}$ and employing Corollary 2, reduces (11) to

$$
\begin{aligned}
\int_{U_{k_{0}, \infty}} \sum a_{i j} D_{i} \phi D_{j} \phi+q \phi^{2} \geqq & \int_{U_{k_{0}, \infty}} \sum a_{i j} D_{i}\left(\frac{\phi^{2} \psi}{u+\varepsilon}\right) D_{j} u+q \phi^{2} \psi \\
& +\int_{U_{k_{0}, \infty}} \frac{\varepsilon q \phi^{2}(1-\psi)}{u+\varepsilon} .
\end{aligned}
$$

Now let $h>0$ be given and choose $\psi$ such that:

$$
\operatorname{supp} \psi \subset\left\{x \mid \operatorname{dist}\left(x, U_{0, k_{0}}\right)<h\right\}, \quad|\operatorname{grad} \psi| \leqq \frac{K}{h} .
$$

Note that the first integral is taken over a subset of $U_{k_{0}, \infty} \cap N_{k_{0}}$ where $u \in C^{1+\alpha}$, and $u>0$ on $R_{k_{0}}$. A simple limit argument as $h \rightarrow 0$, then shows that (12) becomes:

$$
\int_{U_{k_{0}, \infty}} \sum a_{i j} D_{i} \phi D_{j} \phi+q \phi^{2} \geqq-M \int_{R_{k_{0}}} \phi^{2} d s-\int_{U_{k_{0}}, \infty} \frac{|q| \varepsilon}{u+\varepsilon} \phi^{2},
$$

with $M$ a constant independent of $\varepsilon, \phi$. But (compare with [4]), $|q| \phi^{2} \varepsilon /(u+\varepsilon) \rightarrow 0$ a.e. in $U_{k_{0}, \infty}$ and $|q| \phi^{2} \varepsilon /(u+\varepsilon) \leqq|q| \phi^{2} \in L^{1}\left(U_{k_{0}, \infty}\right)$. By the Lebesque Convergence theorem we conclude that

$$
\lim _{\varepsilon \rightarrow 0} \int_{U_{k_{0}, \infty}} \frac{\varepsilon|q| \phi^{2}}{u+\varepsilon}=0
$$

Consequently, (13) becomes:

$$
\int_{U_{k_{0}, \infty}} \sum a_{i j} D_{i} \phi D_{j} \phi+q \phi^{2} \geqq-M \int_{R_{k_{0}}} \phi^{2} d s
$$


But, by assumption (e), we have:

$$
\int_{U_{0, k_{0}}} \sum a_{i j} D_{i} \phi D_{j} \phi+q \phi^{2} \geqq M \int_{R_{k_{0}}} \phi^{2} d s,
$$

for some finite dimensional subspace $F$ and $\phi \in C_{0}^{\infty}(G) \cap F^{\perp}$. Our result follows by combining (14) and (15).

As a final result we state:

CoROLlaRy 3. Let $L$ denote a regularly accretive operator defined from $B$ on $C_{0}^{\infty}(G)$ (see [10]), and let $S(L)$ denote the spectrum of $L$. Then $S(L) \cap(-\infty, 0)$ is finite.

\section{REFERENCES}

1. S. Agmon, The $L_{p}$ approach to the Dirichlet problem, Annali Scuola Norm. Sup. Pisa, 13 (1959), 405-448.

2. W. Allegretto, On the equivalence of two types of oscillation for elliptic operators, Pacific J. Math., 55 (1974), 319-328.

3. - Spectral estimates and oscillation of singular differential operators, Proc. Amer. Math. Soc., (to appear).

4. - A comparison theorem for nonlinear operators, Annali Scuola Norm. Sup. Pisa, 25 (1971), 41-46.

5. A. Friedman, Partial Differential Equations, Holt, Rinehart and Winston, Inc., New York, 1969.

6. K. Kreith, Oscillation Theory, Lecture Notes in Mathematics, Vol.324, SpringerVerlag, 1973.

7. W. Moss and J. Piepenbrink, Positive solutions of elliptic equations, Pacific J. Math., 75 (1978), 219-226.

8. J. Piepenbrink, Nonoscillatory elliptic equations, J. Differential Equations, 15 (1974), 541-550.

9. — A conjecture of Glazman, J. Differential Equations, 24 (1977), 173-177.

10. M. Schechter, Spectra of Partial Differential Operators, North Holland, Amsterdam, 1971.

11. G. Stampacchia, Équations Elliptiques du Second Ordre à Coefficients Discontinus, Les Presses de l'Universite de Montreal, 1966.

12. C. A. Swanson, Comparison and Oscillation Theory of Linear Differential Equations, Mathematics in Science and Engineering, Vol. 48, Academic Press, New York, 1968.

Received April 11, 1979.

UNIVERSITY OF ALBERTA

Edmonton, Alberta T6G 2G1, Canada 



\section{PACIFIC JOURNAL OF MATHEMATICS}

\section{EDITORS}

DONALD BABBITT (Managing Editor)

University of Galifornia

Los Angeles, California 90024

Hugo RossI

University of Utah

Salt Lake City, UT 84112

C. C. MOORE AND ANDREW OGG

University of California

Berkeley, CA 94720
J. DUGUNDJI

Department of Mathematics University of Southern California Los Angeles, California 90007

R. Finn AND J. Milgram Stanford University Stanford, California 94305

\section{ASSOCIATE EDITORS}

R. ARENS

E. F. BECKENBACH

B. H. NEUManN

F. WOLF

K. YOSHIDA

\section{SUPPORTING INSTITUTIONS}

UNIVERSITY OF ARIZONA

UNIVERSITY OF BRITISH COLUMBIA CALIFORNIA INSTITUTE OF TECHNOLOGY

UNIVERSITY OF CALIFORNIA

MONTANA STATE UNIVERSITY

UNIVERSITY OF NEVADA, RENO

NEW MEXICO STATE UNIVERSITY

OREGON STATE UNIVERSITY
UNIVERSITY OF OREGON

UNIVERSITY OF SOUTHERN CALIFONIA

STANFORD UNIVERSITY

UNIVERSITY OF HAWAII

UNIVERSITY OF TOKYO

UNIVERSITY OF UTAH

WASHINGTON STATE UNIVERSITY

UNIVERSITY OF WASHINGTON 


\section{Pacific Journal of Mathematics}

\section{Vol. 92, No. $1 \quad$ January, 1981}

Michael E. Adams and J. Sichler, Lattices with unique complementation . ....1

Walter Allegretto, Positive solutions and spectral properties of second order

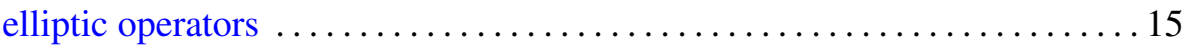

Philip J. Boland and Sean Dineen, Holomorphy on spaces of distribution . . 27

Duncan Alan Buell, Philip A. Leonard and Kenneth S. Williams, Note on

the quadratic character of a quadratic unit $\ldots \ldots \ldots \ldots \ldots \ldots \ldots \ldots \ldots$

Herbert Busemann and Bhalchandra B. Phadke, Two theorems on

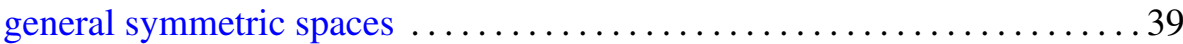

Emeric Deutsch, Bounds for the Perron root of a nonnegative irreducible

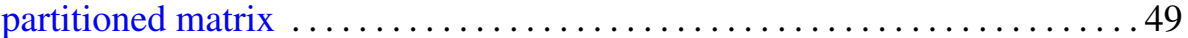

Charles F. Dunkl, A difference equation and Hahn polynomials in two

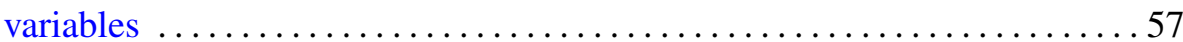

Gustave Adam Efroymson, The Riemann mapping theorem for planar Nash

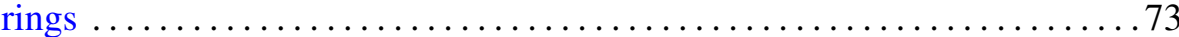

John Albert Fridy and Robert Ellis Powell, Tauberian theorems for matrices generated by analytic functions

Denton Elwood Hewgill, John Hamilton Reeder and Marvin Shinbrot,

Some exact solutions of the nonlinear problem of water waves .......887

Bessie Hershberger Kirkwood and Bernard Robert McDonald, The

symplectic group over a ring with one in its stable range

Esther Portnoy, Transitive groups of isometries on $H^{n}$

Jerry Ridenhour, On the sign of Green's functions for multipoint boundary

value problems

Nina M. Roy, An $M$-ideal characterization of $G$-spaces

Edward Barry Saff and Richard Steven Varga, On incomplete

polynomials. II

Takeyoshi Satō, The equations $\Delta u=P u(P \geqq 0)$ on Riemann surfaces and

isomorphisms between relative Hardy spaces

James Henry Schmerl, Correction to: "Peano models with many generic classes"

Charles Madison Stanton, On the closed ideals in $A(W)$

Viakalathur Shankar Sunder, Unitary equivalence to integral operators

Pavel G. Todorov, New explicit formulas for the $n$th derivative of composite functions

James Li-Ming Wang, Approximation by rational modules on boundary sets

Kenneth S. Williams, The class number of $Q(\sqrt{p})$ modulo 4 , for $p \equiv 5$

$(\bmod 8)$ a prime 